\title{
CCAT2 is an oncogenic long non-coding RNA in pancreatic ductal adenocarcinoma
}

\author{
Yi Cai ${ }^{1}$, Xiaomei Li ${ }^{1}$, Peng Shen ${ }^{2}$ and Dong Zhang ${ }^{1 *}$
}

\begin{abstract}
Background: Pancreatic ductal adenocarcinoma (PDAC) is highly aggressive with poor prognosis. Long non-coding RNAs (IncRNAs), a group of non-coding RNAs, play important roles in the progression of PDAC. This study aimed to investigate the potential involvement of InCRNA CCAT2 in PDAC tumorigenesis.

Methods: Expression of CCAT2 was detected by quantitative real-time PCR (qRT-PCR) in 80 human PDAC tissues and three PDAC cell lines. The effects of CCAT2 silencing in PANC-1 cells on cell proliferation and invasion were studied using MTT assay and transwell assay, respectively. The effect of CCAT2 silencing on tumorigenesis was assessed by PANC-1 xenograft in vivo. Using si-KRAS, the role of KRAS to regulate CCAT2 was evaluated by qRT-PCR and luciferase reporter assay. The involvement of MEK/ERK and PI3K/AKT signaling in CCAT2 regulation was investigated by pathway inhibitors PD98059 and LY294002, respectively.
\end{abstract}

Results: CCAT2 was significantly elevated in high-grade PDAC tissues and higher CCAT2 expression was correlated with lower survival rate in PDAC patients. CCAT2 was up-regulated in PDAC cell lines, as compared with normal pancreatic cells. Silencing of CCAT2 inhibited cell proliferation and invasion in PANC-1 cells in vitro, and attenuated tumorigenesis of PANC-1 xenograft in vivo. Furthermore, CCAT2 was regulated by KRAS through MEK/ERK signaling pathway.

Conclusions: CCAT2 is an oncogenic InCRNA in PDAC likely regulated by the KRAS-MEK/ERK pathway. It could be a potential diagnostic biomarker and therapeutic target for PDAC.

Keywords: PDAC, IncRNA CCAT2, KRAS, MAPK signaling

\section{Background}

Pancreatic ductal adenocarcinoma (PDAC), which derives from the epithelial cells of pancreatic duct, is the predominant form of pancreatic cancer [1,2]. Despite tremendous efforts to understand the pathogenesis and to improve diagnostic and therapeutic strategies of PDAC, it remains to be an incurable lethal disease with less than $5 \%$ of overall 5 -year survival rate.

More than $90 \%$ of PDAC carry activating KRAS mutations, which are initiating genetic alterations of this disease [3]. The KRAS proto-oncogene encodes for the KRAS protein. KRAS, as a small GTPase, couples various

\footnotetext{
*Correspondence: zhangdong_301@163.com

1 Department of Geriatric Oncology, The General Hospital of Chinese People's Liberation Army, 28 Fuxing Road, Haidian District, Beijing 100853, People's Republic of China

Full list of author information is available at the end of the article

Yi Cai and Xiaomei Li are the co-first authors.
}

growth-factor receptors on the cell membrane to intracellular signaling pathways and transcription factors, thus controlling diverse cellular processes [4]. Most KRAS mutations impairs intrinsic GTPase activity of KRAS, resulting in an aberrant protein that is constitutively activating downstream oncogenic signaling pathways, including PI3K/AKT and MEK/ERK [5]. Meanwhile, aberrant KRAS constantly activates a wide range of transcription factors, promoting cell proliferation, survival, transformation, adhesion, and migration [6]. Although KRAS signaling is perceived as the major driving force of PDAC [7], intensive effort to explore KRAS as effective therapeutic target in PDAC has largely failed to reach the clinic [8]. Therefore, there is an urgent need to develop alternative strategies to effectively target KRAS signaling, such as blocking KRAS downstream pathways, KRAS downstream effectors or KRAS upstream modulators. 
Recent studies have suggested that long non-coding RNAs (lncRNAs) (> 200 nucleotides in length), one of the two widely-investigated classes of non-coding RNAs, plays essential roles in the development, progression, drug resistance, and epigenetic modification of PDAC [9]. Importantly, several lncRNAs closely correlate with KRAS signaling in PDAC. For instance, lncRNA MIR31HG competes with KRAS for miR-193b binding site, therefore abolishing the inhibitory effect of miR$193 b$ on KRAS and promoting PDAC progression [10]. lncRNA MALAT1 could sequester miR-217 via direct binding, thus protecting KRAS from miR-217-mediated degradation and inducing PDAC proliferation [11, 12]. Nevertheless, the underlying mechanisms of lncRNAmediated regulation in KRAS signaling or PDAC remain unclear.

LncRNA CCAT2, located at $8 \mathrm{q} 24$, is recently identified from microsatellite-stable colorectal cancer. It induces tumor growth, metastasis, chromosomal instability, and is considered as an oncogenic lncRNA [13]. Further studies confirmed the involvement of CCAT2 in the tumorigenesis of many other cancers, including cervical cancer $[14,15]$, bladder cancer [16], ovarian cancer [17], hepatocellular carcinoma [18], glioma [19], gastric cancer [20] and breast cancer [21]. Here, we aimed to investigate the expression of CCAT2 in human PDAC tissues and PDAC cell lines, to determine the functions of CCAT2 in PDAC in vitro and in vivo, and to explore the involvement of CCAT2 in KRAS signaling in PDAC.

\section{Methods}

\section{Human PDAC tissues collection}

A total of 80 PDAC patients under pancreaticoduodenal resection were enrolled from The General Hospital of Chinese People's Liberation Army between March 2007 and October 2015. Clinical characteristics of these patients were summarized in Table 1 . The resected PDAC tissues were fixed in formalin and embedded in paraffin for pathological diagnosis, or snap-frozen immediately in liquid nitrogen for RNA extraction.

\section{HE staining}

The pathological tissues were fixed in normalized fixative, consisting of $4 \%$ paraformaldehyde in $0.01 \mathrm{M}$ phosphate-buffered saline, overnight at room temperature. The tissue blocks were then dehydrated with an ascending ethanol series, cleared with xylene and then embedded in paraffin. The paraffin blocks were cut into transverse serial sections of $10 \mu \mathrm{m}$ thickness. Next, five sections from each animal were randomly chosen and mounted on poly-L-lysine coated slides for HE staining. The detailed protocol was shown below: (1) Deparaffinize sections, 2 changes of xylene, 10 min each. (2) Re-hydrate
Table 1 Correlations between CCAT2 expression and clinicopathologic variables in $\mathbf{8 0}$ cases of human PDAC

\begin{tabular}{|c|c|c|c|c|}
\hline \multirow[t]{2}{*}{ Characteristics } & \multirow[t]{2}{*}{ No. of patients } & \multicolumn{2}{|c|}{$\begin{array}{l}\text { CCAT2 expression } \\
\text { [case (\%)] }\end{array}$} & \multirow[t]{2}{*}{$P$ value } \\
\hline & & Low & High & \\
\hline \multicolumn{5}{|l|}{ Age (years) } \\
\hline$<60$ & 43 & 48.8 & 51.2 & 0.426 \\
\hline$>60$ & 37 & 42.2 & 56.8 & \\
\hline \multicolumn{5}{|l|}{ Gender } \\
\hline Male & 47 & 53.2 & 46.8 & 0.411 \\
\hline Female & 33 & 42.4 & 57.6 & \\
\hline \multicolumn{5}{|l|}{ Tumor size (cm) } \\
\hline$<4$ & 31 & 61.3 & 38.7 & 0.078 \\
\hline$\geq 4$ & 49 & 44.9 & 55.1 & \\
\hline \multicolumn{5}{|l|}{ Histologic grade } \\
\hline G1 or G2 & 35 & 68.6 & 31.4 & 0.004 \\
\hline G3 & 45 & 26.7 & 73.3 & \\
\hline \multicolumn{5}{|l|}{ pT category } \\
\hline $\mathrm{T} 1$ or $\mathrm{T} 2$ & 31 & 67.7 & 33.3 & 0.007 \\
\hline $\mathrm{T} 3$ or T4 & 49 & 20.4 & 79.6 & \\
\hline
\end{tabular}

in 2 changes of absolute alcohol, 5 min each. (3) 95\% alcohol for 2 min and 70\% alcohol for 2 min. (4) Wash briefly in distilled water. (5) Stain in Harris hematoxylin solution for $8 \mathrm{~min}$. (6) Wash in running tap water for $5 \mathrm{~min}$. (7) Differentiate in $1 \%$ acid alcohol for $30 \mathrm{~s}$. (8) Wash running tap water for $1 \mathrm{~min}$. (9) Bluing in $0.2 \%$ ammonia water or saturated lithium carbonate solution for $30 \mathrm{~s}$ to $1 \mathrm{~min}$. (10) Wash in running tap water for $5 \mathrm{~min}$. (11) Rinse in 95\% alcohol, 10 dips. (12) Counterstain in eosin-phloxine solution for $30 \mathrm{~s}$ to $1 \mathrm{~min}$. (13) Dehydrate through 95\% alcohol, 2 changes of absolute alcohol, 5 min each. (14) Clear in 2 changes of xylene, 5 min each. (15) Mount with xylene based mounting medium.

\section{Cell lines and chemicals}

Human pancreatic cancer cell line PANC-1, SW1990, PC-3 and human normal pancreatic ductal epithelial cell line HPDE6-C7 were purchased from Beijing Zhongyuan Ltd. (China). Cells were maintained in DMEM supplemented with $10 \%$ fetal bovine serum (Gibco, Life Technologies, Carlsbad, CA, USA) at $37{ }^{\circ} \mathrm{C}$ under $5 \% \mathrm{CO}_{2}$ in a humidified incubator. The MEK/ERK signaling inhibitor PD98059 [22] and PI3K/AKT signaling inhibitor LY294002 [23] were purchased from Sigma Ltd. (Shanghai, China).

\section{siRNA transfection}

All siRNAs were commercially constructed by Shanghai GenePharma Co. Ltd (Shanghai, China) and transfected with Lipofectamine RNAiMAX reagent (Thermo 
Fisher Scientific, Sunnyvale, CA, USA) according to the manufacturer's protocol. Sequences for siRNA targeting CCAT2 (si-CCAT2) were 5'-GUGCAACUCUGCAAUUUAAUU- $3^{\prime}$ (S) and 5'-UUAAAUUGCAGAGUUGCACUU-3' (AS); Sequences for siRNA targeting KRAS (si-KRAS) were 5'-AUAUUCAGUCAUUUUCAGCAG-3' (S) and 5'-GCUGAAAAUGACUGAAUAUAA-3' (AS). A scramble siRNA (Scramble) was used as negative control, the sequences for Scramble were $5^{\prime}$-GUAAUUUAAGCAACUCUGCUU-3' (S) and 5'-UUAGUUGCACAAAUUGCAGUU-3' (AS)

\section{RNA extraction and quantitative RT-PCR}

RNA isolation, reverse transcription and qRT-PCR were performed as described previously [21] with minor modification. Total RNA was isolated using TRIzol reagent (Thermo Fisher Scientific, Sunnyvale, CA, USA), and reverse transcribed with SuperScript First Strand cDNA System (Thermo Fisher Scientific, Sunnyvale, CA, USA) according to the manufacturer's instructions. qRT-PCR was performed on a 7300 Real-Time PCR System (Thermo Fisher Scientific, Sunnyvale, CA, USA) using SYBR green agent (Applied Biosystem, Foster City, CA, USA). The cycling conditions were 40 cycles of $94{ }^{\circ} \mathrm{C}$ for $30 \mathrm{~s}, 60{ }^{\circ} \mathrm{C}$ for $30 \mathrm{~s}$ and $72{ }^{\circ} \mathrm{C}$ for $30 \mathrm{~s}$. The primers were: CCAT2 forward (5'-AGACAGTGCCAGCCAACC- $\left.3^{\prime}\right)$ and reverse (5'-TGCCAAACCCTTCCCTTA-3'); GAPDH (internal control) forward (5'-ACCCAGAAGACTGTGGATGG-3') and reverse (5'-TCAGCTCAGGGATGACCTTG-3').

\section{MTT assay}

PANC-1 cells were seeded into 96-well plates at a density of $5 \times 10^{3}$ cells/well and a final volume of $150 \mu \mathrm{L} /$ well in triplicate per experiment. After $0,24,48,72$ or $96 \mathrm{~h}$, MTT reagent (Sigma-Aldrich, Shanghai, China) $(20 \mu \mathrm{L})$ was added and cells were incubated for $4 \mathrm{~h}$ at $37^{\circ} \mathrm{C}$. The medium was then discarded and cells were oscillated in $150 \mu \mathrm{L} /$ well dimethyl sulfoxide for $15 \mathrm{~min}$. The absorbance was measured at $490 \mathrm{~nm}$ using a Fluoroskan Ascent FL Microplate Fluorometer (Thermo Scientific, Sunnyvale, CA, USA).

\section{Transwell assay}

Cell motility was evaluated with the 24-well Boyden chamber with $8-\mu \mathrm{m}$ pore size polycarbonate membrane (Corning Incorporated, Los Angeles, CA, USA) with matrigel (BD Biosciences, San Diego, CA, USA) to simulate matrix barrier. $48 \mathrm{~h}$ after siRNA transfection, $4 \times 10^{3}$ cells in $200 \mu \mathrm{L}$ serum-free DMEM medium were seeded on top of the transwell membrane in the upper chamber. $600 \mu \mathrm{L}$ of DMEM medium containing 20\% fetal bovine serum or equal volume of migration buffer was added in the lower chamber as chemo-attractant or negative control, respectively. After 24-h incubation, the membranes were fixed with methanol and stained with $0.1 \%$ crystal violet at $37^{\circ} \mathrm{C}$. Three visual fields were randomly selected from each membrane, and the number of migrated cells were counted underneath an inverted microscope.

\section{Tumor xenograft model}

Female athymic nude mice ( $4-5$ weeks old) were purchased from Vital River Laboratory Animal Technology Ltd. (Beijing, China). $2 \times 10^{6}$ PANC-1 cells, transfected with si-CCAT2 or Scramble, were mixed with equal volume of matrigel (BD Biosciences, USA) and injected subcutaneously into the right flank of nude mice to establish PDAC xenograft models [21]. The perpendicular diameters of all tumors were measured once a week with a digital caliper and the tumor volumes were calculated as (length $\times$ width $\left.^{2}\right) / 2$. All mice were sacrificed at the end of week 4 to compare tumor growth. The animal protocol was approved by the Institutional Animal Care and Use Committee of the General Hospital of Chinese People's Liberation Army.

\section{Western blotting}

Cell lysates were harvested and protein concentrations were determined via bicinchoninic acid protein quantification method [24]. $40 \mu \mathrm{g}$ of total protein were separated by SDS-PAGE gel electrophoresis and electrotransferred onto PVDF membranes. Primary rabbit antibodies against total ERK (No. 4695), p-ERK (No. 4370), total AKT (No. 4685), p-AKT (No. 4060), KRAS (No. 3339) (Cell Signaling Technology Inc., Berkeley, CA, USA) and GAPDH (No. TA-08) (loading control) (Zhongshanjinqiao Biotech, China) were incubated at $4{ }^{\circ} \mathrm{C}$ overnight at a dilution of 1:1000, and after washed with PBST for three times, the secondary horseradish-peroxidase-labeled antibody was incubated at room temperature for $2 \mathrm{~h}$ at a dilution of 1:5000. Finally, the relevant protein was visualized by staining with the enhanced chemiluminescent (ECL) kit (Haigene, Harbin, China). The relative levels of each target protein to the control (total AKT, total ERK, or GAPDH) were determined using a UVP bioimaging system and LabWorks 4.6 software (UVP, Upland, CA, USA).

\section{Luciferase assay}

PANC-1 cells in 24-well plates were cotransfected with a dual-luciferase reporter plasmid containing CCAT2 
promoter-reporter plasmid (Genechem Ltd., Shanghai, China), in combination with si-KRAS or Scramble for $48 \mathrm{~h}$. The luciferase activity was measured using the Dual-Lucy Assay Kit from Vigorous Biotechnology (Beijing, China) according to the manufacturer's protocol. All transfections were repeated for at least three times.

\section{Statistical analysis}

All statistical analyses were carried out using SPSS 17.0 (SPSS Inc., USA). The overall survival was evaluated by the Kaplan-Meier method. All other data were analyzed using independent two-tailed Student's $t$ test. Data shown were mean \pm SEM or representative of at least three independent biological repeats. $\mathrm{P}<0.05$ was considered as statistically significant.

\section{Results}

CCAT2 is upregulated in PDACs and predicts patients with poor survival

We first categorized 80 human PDAC tissue samples into low-grade $(\mathrm{n}=35)$ or high-grade $(\mathrm{n}=45)($ Fig. 1a), and used qRT-PCR to detect their endogenous CCAT2 expression. CCAT2 levels were significantly higher in the high-grade PDAC tissues than those in the low-grade PDACs $(\mathrm{P}=0.004)$ (Fig. 1b, Table 1$)$. CCAT2 expression was also significantly related to $\mathrm{pT}$ (primary tumor) categories $(\mathrm{P}=0.007, \mathrm{~T} 1+\mathrm{T} 2$ vs $\mathrm{T} 3+\mathrm{T} 4)$ (Table 1$)$. However, there was no correlation between CCAT2 expression and age, gender, or tumor size in these 80 PDAC patients (Table 1). A Kaplan-Meier survival curve showed that the overall survival rate of PDAC patients in high CCAT2 expression group $(\mathrm{n}=44)$ markedly

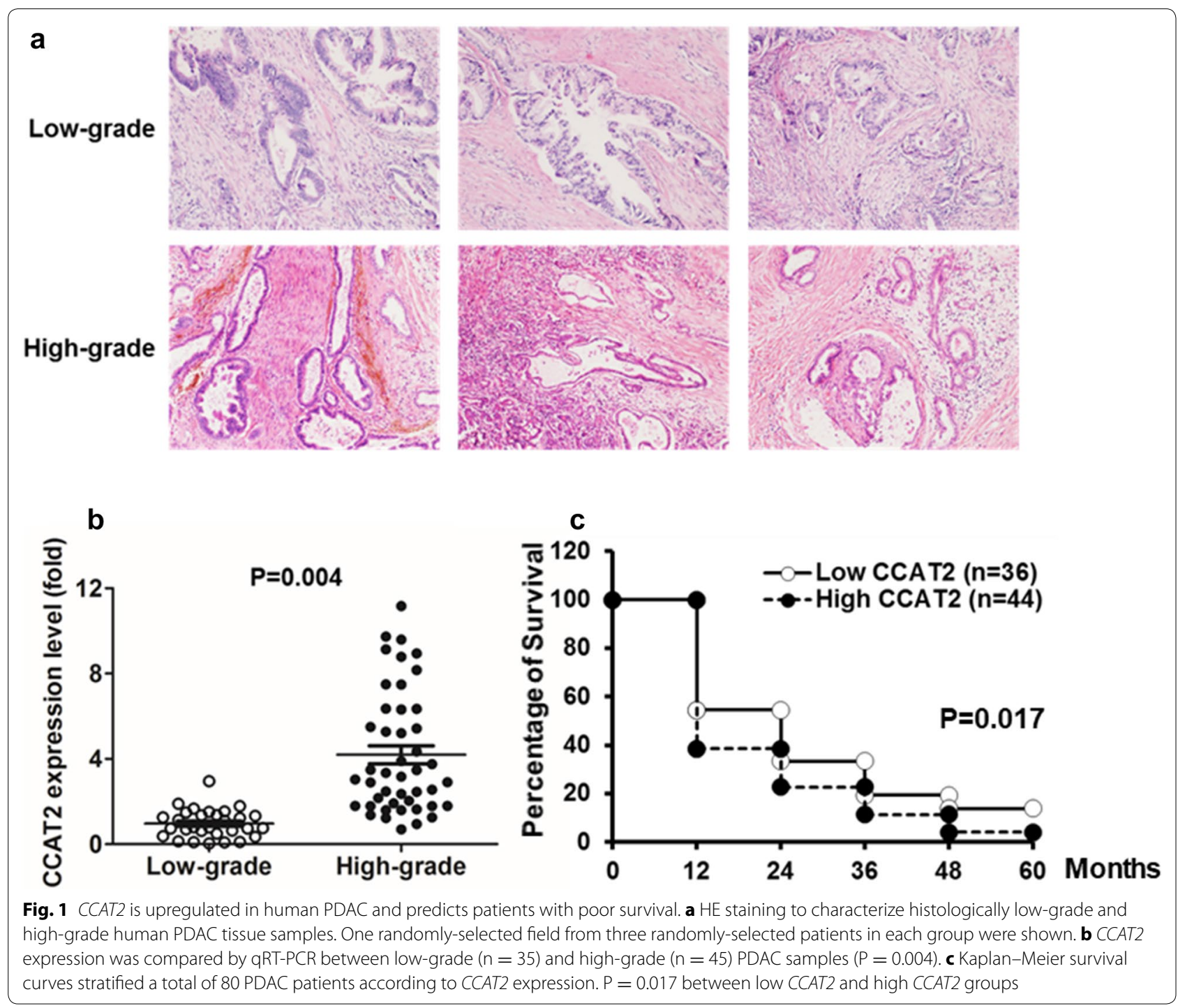


decreased as compared with that of low CCAT2 expression $(\mathrm{n}=36)$ group (Fig. 1c).

\section{Silencing of CCAT2 inhibits PDAC cell proliferation} and invasion in vitro

Compared with HPDE6-C7, a normal human pancreatic ductal epithelial cell line, we found higher CCAT2 expression in all three PDAC cell lines, including PANC-1
$(\mathrm{P}<0.01)$, SW1990 $(\mathrm{P}<0.01)$ and $\mathrm{PC}-3(\mathrm{P}<0.05)$ (Fig. 2a). Next, we knocked down the expression of endogenous CCAT2 in PANC-1 cells via CCAT2 siRNA (si-CCAT2) to investigate its role in proliferation and invasion of PDACs (Fig. 2b). CCAT2 silencing dramatically inhibited the proliferation of PANC-1 cells at $72(\mathrm{P}<0.05)$ and $96(\mathrm{P}<0.05)$ hours post-transfection (Fig. 2c). Furthermore, compared with Scramble-treated cells, the capability of invasion was

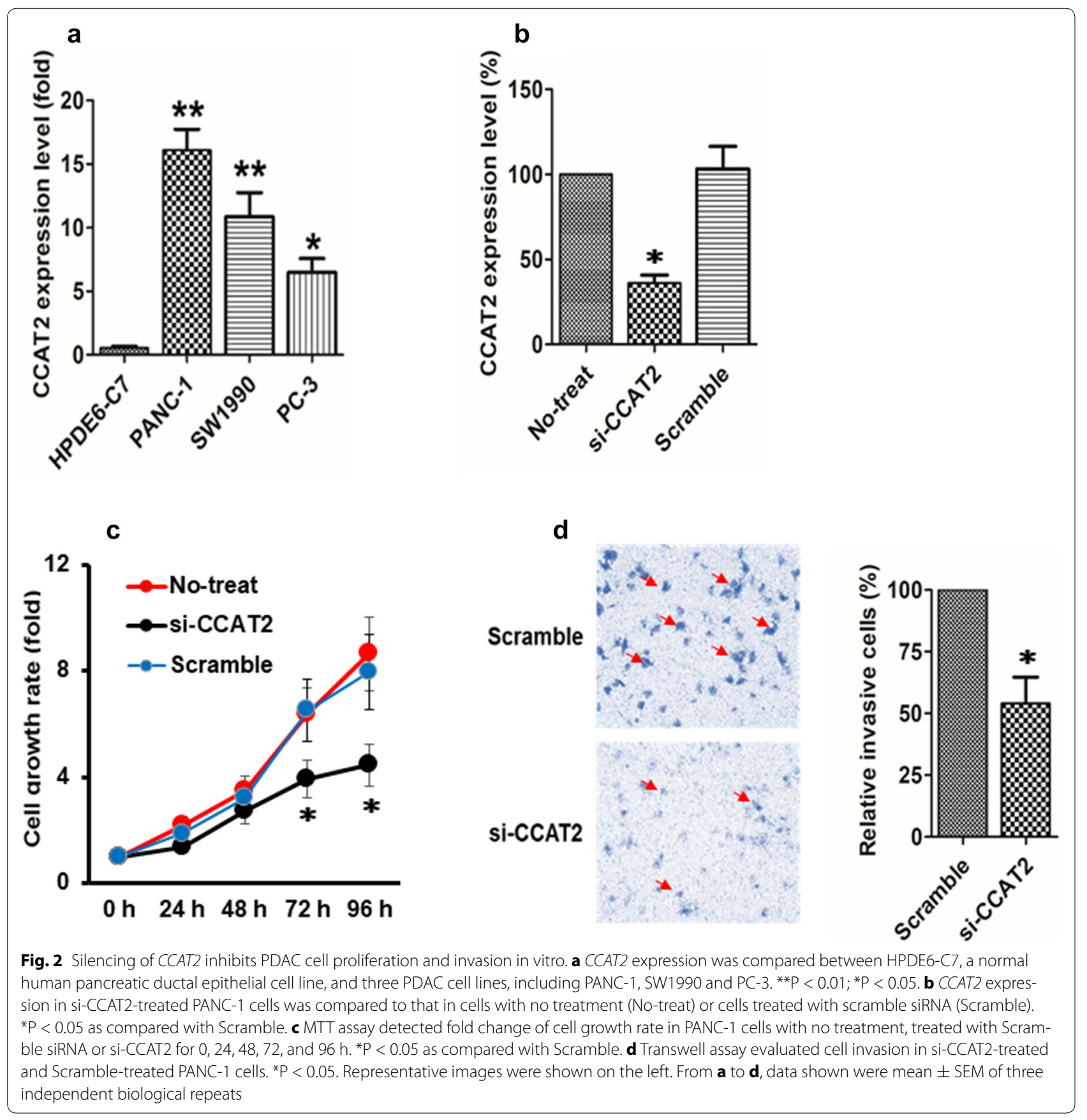


significantly suppressed in PANC-1 cells transfected with si-CCAT2 $(P<0.05)$ (Fig. 2d).

\section{Silencing of CCAT2 inhibits PDAC tumor growth in vivo} To explore potential involvement of CCAT2 in the development of PDAC, we established PDAC xenograft mouse models with PANC-1 cells transfected with either Scramble $(\mathrm{n}=5)$ or si-CCAT2 $(\mathrm{n}=5)$. Silencing of CCAT2 $(\mathrm{P}<0.01)$ (Fig. 3a) significantly reduced PDAC tumor growth at $3(\mathrm{P}<0.05)$ and 4 weeks $(\mathrm{P}<0.05)$ after xenograft injections (Fig. 3b). Consistently, we found tumor size (Fig. 3c) and weight (Fig. 3d) were dramatically suppressed in the si-CCAT2 group, compared with those in the Scramble group. These results suggest that $C C A T 2$ contributes to PDAC tumorigenesis in vivo.

\section{KRAS regulates CCAT2 expression via MEK/ERK pathway} Other studies have revealed that the oncogenic KRAS mutation is present in more than $90 \%$ of PDAC and is the driving force of pancreatic tumorigenesis [25], we showed in this study that suppression of KRAS expression (Fig. 4a) led to a significant downregulation of CCAT2 expression in PANC-1 cells (Fig. 4b). Additionally, KRAS inhibition markedly decreased CCAT2 promoter activity by more than $60 \%$ (Fig. $4 \mathrm{~d}$ ), indicating that KRAS might regulate CCAT2 expression at transcriptional level. Although inhibition of KRAS attenuated the activation of both MEK/ERK and PI3K/AKT signaling pathways (Fig. 4c), we found that CCAT2 expression was only suppressed by MEK/ERK pathway inhibitor PD98059, but not PI3K/AKT pathway inhibitor LY294002 (Fig. 4e, f). Altogether, our data indicate that
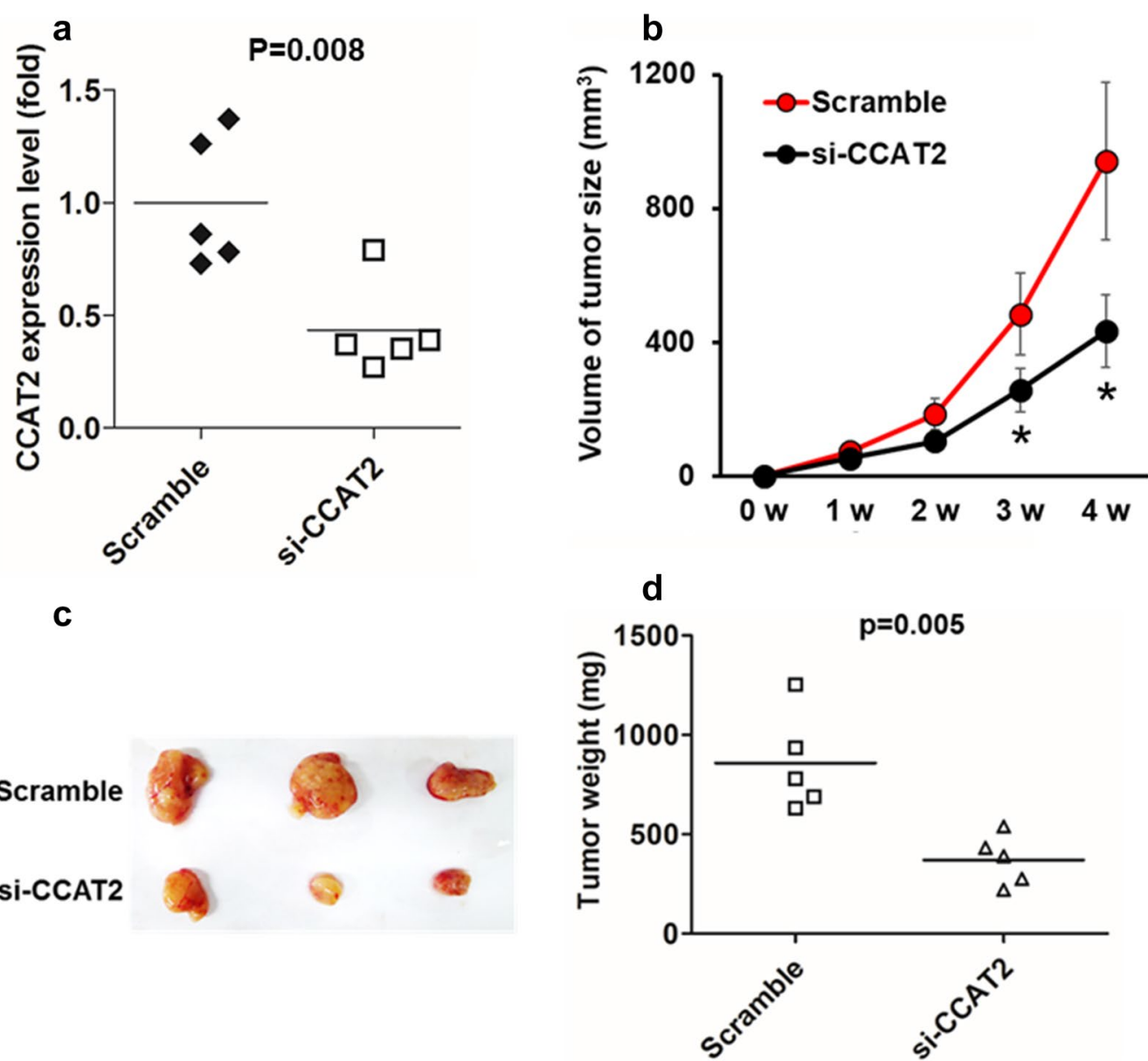

Fig. 3 Silencing of CCAT2 inhibits PDAC tumor growth in vivo. Female nude mice ( $\mathrm{n}=5 / \mathrm{group}$ ) was implanted with si-CCAT2-treated or Scrambletreated PANC-1 xenografts and tumor growth was monitored continuously for 4 weeks. a CCAT2 expression in xenograft PDAC tumors was compared between si-CCAT2 and Scramble groups $(P=0.008)$ when tumors were harvested 4 weeks after PANC-1 cell injection. b Tumor volume was recorded once per week after PANC-1 cells injection. ${ }^{*} P<0.05$. $\mathbf{c}$ Representative images of PDAC xenograft tumors from each group at the time of study termination (week 4). d Tumor weight were compared between si-CCAT2-treated or Scramble-treated groups $(P=0.005)$ 


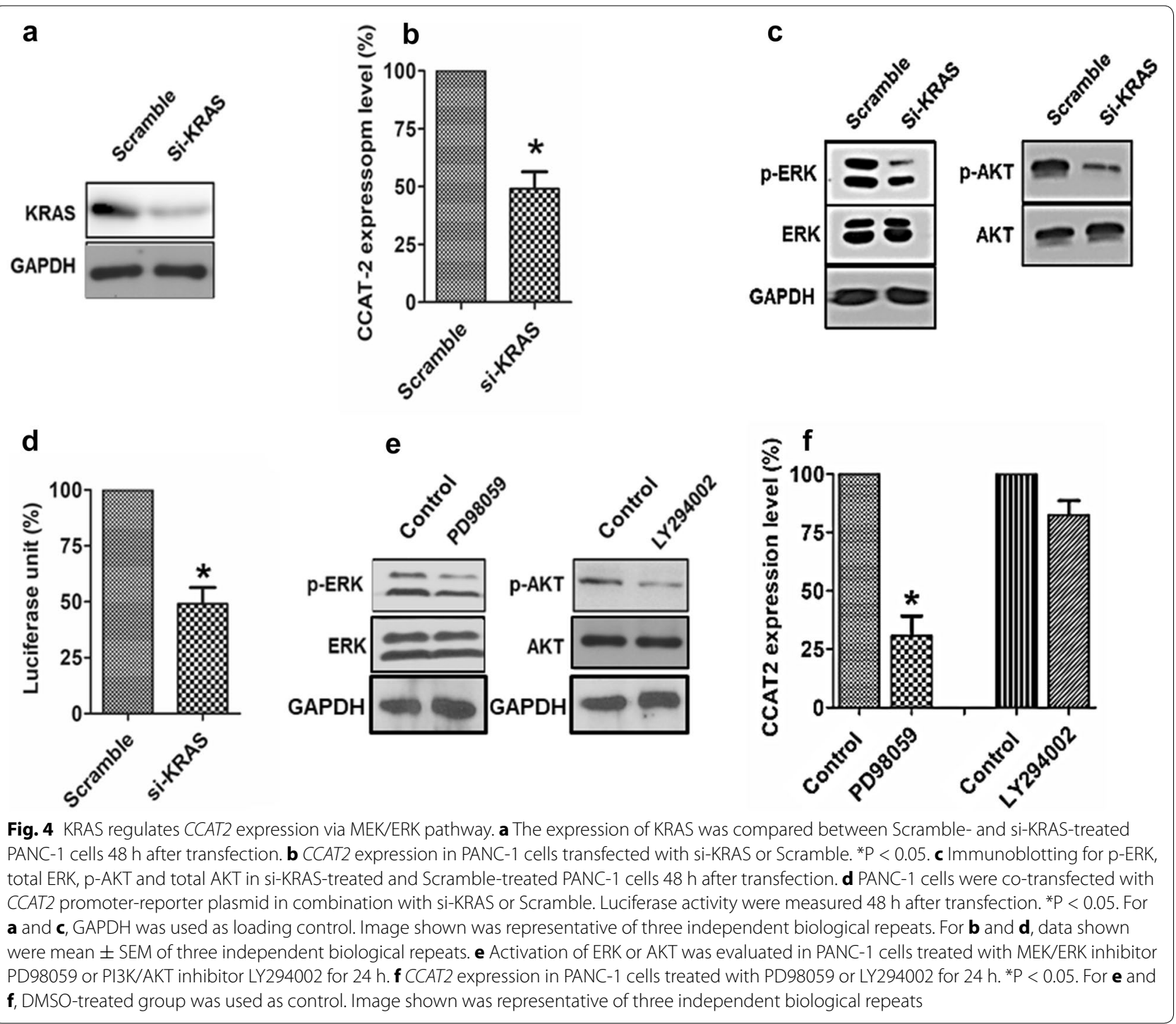

KRAS might transcriptionally regulate CCAT2 expression via MEK/ERK signaling pathway in PDAC.

\section{Discussion}

PDAC is one of the most aggressive malignancies. Because of local invasion and distal metastasis, less than $20 \%$ of PDAC is resectable at the time of diagnosis [26]. Lack of effective biomarkers in PDAC further compromises the early diagnosis and treatment of this disease. Increased expression of CCAT2 has been found in a wide range of cancers, promoting tumor growth, cell cycle progression, migration, invasion, metastasis, and inhibiting apoptosis [13-21], however, the function of CCAT2 in PDAC was still unknown.

In this study, we found for the first time that lncRNA CCAT2 was significantly elevated in human high-grade
(G3) PDAC tissues, as compared with low-grade (G1 or G2) tissues. The differential expression of CCAT2 in PDAC is not age- or gender-related. Intriguingly, CCAT2 expression is closely correlated with mortality of PDAC patients in that higher CCAT2 levels predicts lower survival rate. Therefore, it could be promising to explore CCAT2 as a universal predictive biomarker in PDAC, in order to benefit patients' quality of life and prognosis. Furthermore, using PANC-1 as a PDAC cell model, we showed that inhibition of CCAT2 significantly decreased cell proliferation and invasion in vitro, and suppressed tumorigenicity in vivo. These data suggest that CCAT2 is oncogenic in PDAC.

Several mechanisms underlying the tumorigenic function of CCAT2 have been proposed. CCAT2 could activate the transcriptional activity of $\mathrm{Wnt} / \beta$-catenin 
signaling pathway and promote the translocation of $\beta$-catenin from cytoplasm to nucleus $[19,21]$; CCAT2 could induce chromosomal instability, causing aneuploidy formation [13]; CCAT2 could up-regulate the expression of $M Y C$ oncogene and its microRNA target miR-17-5p [13]; CCAT2 could decrease E-cadherin and increase ZEB2, Vimentin, and N-cadherin, thus stimulating epithelial-mesenchymal transition (EMT) [27]. The detailed mechanism of how CCAT2 facilitates the development of PDAC need to be further explored in our future studies.

The expression of IncRNA is under tight control. For example, lncRNA MEG3 is decreased by hypermethylation in its promoter [28]; lncRNA $L E T$ is repressed by HDAC3-mediated deacetylation in its promoter [29]; lncRNA AK019103 contains binding sites of transcription factor NF- $\mathrm{KB}$, and inhibition of NF- $\mathrm{kB}$ dramatically suppressed DNA damage-induced AK019103 upregulation [30]; lncRNA HOTAIR is inhibited by miRNA-34a via direct binding [31]; IncRNA $U C A 1$ could bind to RNA-binding protein hnRNP I, which in turn stabilizes $U C A 1$ [32]. In this study, we revealed a novel regulatory mechanism of CCAT2's oncogenic potential in PDAC. Our data support that KRAS oncogene induces the expression of CCAT2 via MEK/ERK, but not PI3 K/AKT signaling. Although our data are not sufficient to identify the exact factor directly regulating CCAT2 expression in PDAC, based on the diverse functions in each tier of KRAS or MEK/ERK signaling cascade, we speculate that they could modulate CCAT2 through DNA methylation, histone modification, downstream transcription factors, downstream miRNAs, or post-transcriptional regulations.

In summary, elevated expression of CCAT2 is correlated with high-grade and low survival rate in PDAC. CCAT2 facilitates proliferation and invasion of tumor cells, thus promoting PDAC progression. CCAT2 serves as a downstream effector of KRAS and MEK/ERK signaling, and holds potential to be a novel diagnostic biomarker and a therapeutic target in PDAC. Besides comparatively small sample size (80 PDAC patients were enrolled), another limitation of our study is that we did not compare the expression of CCAT2 between normal pancreatic and PDAC tissues. However, these will be the subject of our ongoing studies.

\footnotetext{
Authors' contributions

$C Y$ and ZD conceived and designed the experiments. CY and LXM performed the experiments. $C Y$ and $L X M$ analyzed the data. $C Y$ and $L X M$ contributed reagents/materials/analysis tools. CY and ZD wrote the manuscript. All authors read and approved the final manuscript.

\section{Author details}

1 Department of Geriatric Oncology, The General Hospital of Chinese People's Liberation Army, 28 Fuxing Road, Haidian District, Beijing 100853, People's Republic of China. ${ }^{2}$ The Fourth Division of Department of Internal Medicine,
}

Huailai County Hospital, Fuqianddong Rd, Huailai, Zhangjiakou, Beihe 075400, People's Republic of China.

\section{Competing interests}

The authors declare that they have no competing interests.

\section{Consent for publication}

I give my consent for publication on behalf of all authors and patients involved.

\section{Ethics approval and consent to participate}

This study was approved by the Ethical Committee for Clinical Research of The General Hospital of Chinese People's Liberation Army, and written informed consent was received from each enrolled patient.

\section{Funding}

This study was supported by the Clinical Research Support Foundation of General Hospital of Chinese People's Liberation Army (No. 2015FC-TSYS-2015).

\section{Publisher's Note}

Springer Nature remains neutral with regard to jurisdictional claims in published maps and institutional affiliations.

Received: 3 February 2017 Accepted: 15 December 2017

Published online: 03 January 2018

\section{References}

1. Taher MA, Khan ZR, Chowdhury MM, Nur EEM, Chowdhury AK, et al. Pylorus preserving pancreaticoduodenectomy vs. standard Whipple's procedure in case of carcinoma head of the pancreas and periampullary carcinoma. Mymensingh Med J. 2015;24:319-25.

2. Dimastromatteo J, Houghton JL, Lewis JS, Kelly KA. Challenges of pancreatic Cancer. Cancer J. 2015;21:188-93.

3. Lennerz JK, Stenzinger A. Allelic ratio of KRAS mutations in pancreatic cancer. Oncologist. 2015;20:e8-9.

4. Kumar R, Gururaj AE, Barnes CJ. p21-activated kinases in cancer. Nat Rev Cancer. 2006;6:459-71.

5. di Magliano MP, Logsdon CD. Roles for KRAS in pancreatic tumor development and progression. Gastroenterology. 2013;144:1220-9.

6. Ryan DP, Hong TS, Bardeesy N. Pancreatic adenocarcinoma. N Engl J Med. 2014;371:2140-1.

7. Bryant KL, Mancias JD, Kimmelman AC, Der CJ. KRAS: feeding pancreatic cancer proliferation. Trends Biochem Sci. 2014;39:91-100.

8. Cox AD, Fesik SW, Kimmelman AC, Luo J, Der CJ. Drugging the undruggable RAS: mission possible? Nat Rev Drug Discov. 2014;13:828-51.

9. Taucher $\mathrm{V}$, Mangge $\mathrm{H}$, Haybaeck J. Non-coding RNAs in pancreatic cancer: challenges and opportunities for clinical application. Cell Oncol (Dordr). 2016;39:295-318.

10. Ni S, Zhao X, Ouyang L. Long non-coding RNA expression profile in vulvar squamous cell carcinoma and its clinical significance. Oncol Rep. 2016:36:2571-8.

11. Wang X, Li M, Wang Z, Han S, Tang X, et al. Silencing of long noncoding RNA MALAT1 by miR-101 and miR-217 inhibits proliferation, migration, and invasion of esophageal squamous cell carcinoma cells. J Biol Chem. 2015;290:3925-35.

12. LV J, Fan HX, Zhao XP, Lv P, Fan JY, et al. Long non-coding RNA Unigene56159 promotes epithelial-mesenchymal transition by acting as a ceRNA of miR-140-5p in hepatocellular carcinoma cells. Cancer Lett. 2016;382:166-75.

13. Ling H, Spizzo R, Atlasi Y, Nicoloso M, Shimizu M, et al. CCAT2, a novel noncoding RNA mapping to 8q24, underlies metastatic progression and chromosomal instability in colon cancer. Genome Res. 2013;23:1446-61.

14. Chen X, Liu L, Zhu W. Up-regulation of long non-coding RNA CCAT2 correlates with tumor metastasis and poor prognosis in cervical squamous cell cancer patients. Int J Clin Exp Pathol. 2015;8:13261-6.

15. Wu L, Jin L, Zhang W, Zhang L. Roles of long non-coding RNA CCAT2 in cervical cancer cell growth and apoptosis. Med Sci Monit. 2016;22:875-9. 
16. Li J, Zhuang C, Liu Y, Chen M, Zhou Q, et al. shRNA targeting long noncoding RNA CCAT2 controlled by tetracycline-inducible system inhibits progression of bladder cancer cells. Oncotarget. 2016;7:28989-97.

17. Huang S, Qing C, Huang Z, Zhu Y. The long non-coding RNA CCAT2 is upregulated in ovarian cancer and associated with poor prognosis. Diagn Pathol. 2016;11:49.

18. Zhou N, Si Z, Li T, Chen G, Zhang Z, et al. Long non-coding RNA CCAT2 functions as an oncogene in hepatocellular carcinoma, regulating cellular proliferation, migration and apoptosis. Oncol Lett. 2016;12:132-8.

19. Guo H, Hu G, Yang Q, Zhang P, Kuang W, et al. Knockdown of long noncoding RNA CCAT2 suppressed proliferation and migration of glioma cells. Oncotarget. 2016;7:81806-14.

20. Wang YJ, Liu JZ, Lv P, Dang Y, Gao JY, et al. Long non-coding RNA CCAT2 promotes gastric cancer proliferation and invasion by regulating the E-cadherin and LATS2. Am J Cancer Res. 2016;6:2651-60.

21. Cai Y, He J, Zhang D. Long noncoding RNA CCAT2 promotes breast tumor growth by regulating the Wnt signaling pathway. Onco Targets Ther. 2015;8:2657-64

22. Wu Y, Yang Y, Yang P, Gu Y, Zhao Z, et al. The osteogenic differentiation of PDLSCs is mediated through MEK/ERK and p38 MAPK signalling under hypoxia. Arch Oral Biol. 2013;58:1357-68.

23. Fu $X$, Feng J, Zeng D, Ding $Y, Y u$ C, et al. PAK4 confers cisplatin resistance in gastric cancer cells via PI3K/Akt- and MEK/ERK-dependent pathways. Biosci Rep. 2014;34:e00094.

24. Walker JM. The bicinchoninic acid (BCA) assay for protein quantitation. Methods Mol Biol. 1994;32:5-8.
25. Eser S, Schnieke A, Schneider G, Saur D. Oncogenic KRAS signalling in pancreatic cancer. Br J Cancer. 2014;111:817-22.

26. Peixoto RD, Speers C, McGahan CE, Renouf DJ, Schaeffer DF, et al. Prognostic factors and sites of metastasis in unresectable locally advanced pancreatic cancer. Cancer Med. 2015;4:1171-7.

27. Zheng J, Zhao S, He X, Zheng Z, Bai W, et al. The up-regulation of long non-coding RNA CCAT2 indicates a poor prognosis for prostate cancer and promotes metastasis by affecting epithelial-mesenchymal transition. Biochem Biophys Res Commun. 2016;480:508-14.

28. Braconi C, Kogure T, Valeri N, Huang N, Nuovo G, et al. microRNA-29 can regulate expression of the long non-coding RNA gene MEG3 in hepatocellular cancer. Oncogene. 2011;30:4750-6.

29. Yang F, Huo XS, Yuan SX, Zhang L, Zhou WP, et al. Repression of the long noncoding RNA-LET by histone deacetylase 3 contributes to hypoxiamediated metastasis. Mol Cell. 2013;49:1083-96.

30. Wan G, Hu X, Liu Y, Han C, Sood AK, et al. A novel non-coding RNA IncRNA-JADE connects DNA damage signalling to histone $\mathrm{H} 4$ acetylation. EMBO J. 2013:32:2833-47.

31. Chiyomaru T, Yamamura S, Fukuhara S, Yoshino H, Kinoshita T, et al. Genistein inhibits prostate cancer cell growth by targeting miR-34a and oncogenic HOTAIR. PLOS ONE. 2013;8:e70372.

32. Huang J, Zhou N, Watabe K, Lu Z, Wu F, et al. Long non-coding RNA UCA1 promotes breast tumor growth by suppression of p27 (Kip1). Cell Death Dis. 2014;5:e1008.

\section{Submit your next manuscript to BioMed Central and we will help you at every step:}

- We accept pre-submission inquiries

- Our selector tool helps you to find the most relevant journal

- We provide round the clock customer support

- Convenient online submission

- Thorough peer review

- Inclusion in PubMed and all major indexing services

- Maximum visibility for your research

Submit your manuscript at www.biomedcentral.com/submit 